\title{
Amorolfine vs. ciclopirox - lacquers for the treatment of onychomycosis
}

\author{
Katarzyna Tabara, Anna E. Szewczyk, Wojciech Bienias, Agnieszka Wojciechowska, Marta Pastuszka, \\ Magdalena Oszukowska, Andrzej Kaszuba
}

Department of Dermatology, Pediatric Dermatology and Dermatological Oncology, Medical University of Lodz, Lodz, Poland Head of Department: Prof. Andrzej Kaszuba MD, PhD

Postep Derm Alergol 2015; XXXII, 1: 40-45

DOI: 10.5114/pdia.2014.40968

\begin{abstract}
Amorolfine $5 \%$ and ciclopirox $8 \%$ nail lacquers are commonly used in topical treatment of onychomycosis. These formulations may be used alone or in combination with oral antifungal agents. Amorolfine and ciclopirox are valuable therapeutic options, however, their usage in monotherapy should be limited. Proper amorolfine and ciclopirox penetration through the nail plate is provided by transungual drug delivery systems. Although amorolfine and ciclopirox have a different mode of action, they both exhibit a broad antifungal activity. The use of antifungal nail lacquers in combination with oral agents, such as terbinafine and itraconazole, improves efficacy of antifungal therapy.
\end{abstract}

Key words: onychomycosis, amorolfine, ciclopirox, lacquer.

\section{Introduction}

Onychomycosis accounts for about $50 \%$ of all nail diseases [1, 2]. Despite new therapeutic methods it is a common infection and its prevalence increases with age [3-5]. According to the data, $2-13 \%$ of all population are diagnosed with the disease, including about $20 \%$ of patients aged $40-60$ years and up to $50 \%$ of patients at the age of 70 years [6]. Polish epidemiological studies have also found onychomycosis to be an important health problem [7]. The condition affects 4-10 folds more toenails than fingernails, which may be due to slower toenail growth and thus makes fungal invasion easier. Toenail onychomycosis more often affects several nail plates and is more difficult to treat than fingernail onychomycosis due to diminished blood supply in the area, particularly in the elderly [8, 9]. In about $30 \%$ of cases, toenail onychomycosis coexists with foot ringworm, which predisposes to the condition [10]. Yeasts and non-dermatophyte mold infections are diagnosed relatively more commonly in fingernails while dermatophyte infections are more often found in toenails [11].

\section{Treatment of onychomycosis}

Several factors must be considered before choosing therapy for onychomycosis: kind of pathogen, onychomycosis subtype, matrix involvement, number of invaded nails, location (toes or fingers), thickness of nail plate and coexistence of other mycoses with different location. Treatment should not be commenced before mycological confirmation of infection. The aim of the treatment is to eradicate the pathogen (confirmed by negative culture and direct microscopic examination) and regression of symptoms i.e., restoration of normal nail appearance. Currently, there are three main pharmacological strategies - oral treatment, topical treatment and combination therapy. Other therapeutic methods improving local drug absorption (such as iontophoresis, ultrasound), as well as laser or photodynamic therapies may also be used, however, they are still not fully available $[12,13]$. Surgical removal of the diseased nail is not often performed nowadays but chemical avulsion with $40 \%$ urea is done. Bifonazole plus 40\% urea preparation, applied daily on the affected nail under occlusion, is available on the market $[14,15]$.

Address for correspondence: Katarzyna Tabara MD, Department of Dermatology, Pediatric Dermatology and Dermatological Oncology, Medical University of Lodz, 1/5 Kniaziewicza St, 91-347 Lodz, Poland, phone: +48 601465 204, e-mail: kgutfreund@wp.pl Received: 30.09.2013, accepted: 2.01.2014. 
Most treatments for onychomycosis are antifungal systemic drugs. The estimated efficacy is about $50-80 \%$ depending on the drug, progression of onychomycosis and the investigated population. In some cases the effectiveness of oral monotherapy in the treatment of toenail onychomycosis may be even lower [16, 17]. Most commonly used oral drugs are terbinafine, itraconazole and fluconazole, which is mainly indicated in candida infection. Griseofulvin (not available in Poland) may also be used, however, the effectiveness is not very high. Side effects and interaction with other drugs should be considered before instituting systemic treatment $[18,19]$.

Monotherapy of onychomycosis with topical preparations is recommended in the following cases:

- distal subungual onychomycosis (DSO) affecting < 50\% of the nail without matrix area involvement, without presence of yellow streaks along the lateral margin of the nail and without yellow onycholytic areas in the central portion of the nail (dermatophytoma);

- "classical" white superficial onychomycosis (WSO); - onychomycosis due to molds (except for those due to Aspergillus sp.) since they usually do not respond to systemic antifungals;

- patients unwilling or unable to tolerate oral therapy;

- patients requiring maintenance therapy after a course of oral therapy $[13,20]$.

Products specifically indicated for topical therapy are lacquers applied on the nail plate. Leading products include ciclopirox $8 \%$ and amorolfine 5\%.

Combination therapy, combining oral and topical treatments, is thought to be the most effective method. Different schemata are indicated e.g. itraconazole and ciclopirox, terbinafine and ciclopirox, terbinafine and amorolfine, itraconazole and amorolfine, fluconazole and amorolfine. Sequential therapy, using two consecutive oral therapies, is a type of combination therapy [21].

\section{Topical preparations}

As it has already been mentioned, amorolfine and ciclopirox lacquers are most common agents used for topical treatment of onychomycosis. Other substances from the imidazole group such as tioconazole, bifonazole, miconazole and ketoconazole as well as preparations containing butenafine, tolnaftate, vitamin E and bitter orange oil are of limited use [21, 22]. Terbinafine in the form of lacquer is being tested [23].

Traditional forms of topical drugs, such as creams or ointments, are not a good vehicle for active substances due to nail keratin structure different from that of epidermis. Ciclopirox $8 \%$ and amorolfine 5\% lacquers were formulated to provide better penetration of active substances through the nail plate and thus increase effectiveness of the agent. Lacquer inactive substances (vehicles) maintain proper concentration of the active substance on the nail surface. Proper drug penetration through the surface of the nail plate is provided by transungual drug delivery systems (TUDDS), which usually contain water-insoluble polymers removed with organic solvents or a newer system with hydroxypropyl chitosan $(\mathrm{HPCH})$ in a water-alcohol solution, water-soluble. Hydroxypropyl chitosan was derived from the exoskeletons of crabs, the substance is characterized by high plasticity and affinity to keratin $[20,24]$. Hydroxypropyl chitosan keeps shine to a minimum and creates a velvety smooth appearance on the surface of the nail, the preparation may also be applied on the area around the nail. One of ciclopirox preparations is formulated on the HPCH vehicle [24].

Concentration of ciclopirox and amorolfine in lacquer increases with solvent evaporation. The film on the nail plate surface enables longer contact of the active substance, improves both nail hydration and agent diffusion through the affected surface $[25,26]$.

\section{Amorolfine}

Amorolfine is a morpholine derivative with antifungal and fungistatic activity, introduced in 1981. It inhibits ergosterol synthesis on two levels: by inhibiting delta 14 reductase and delta 7-8 isomerase, which affects pathogen membrane synthesis, depletes ergosterol and causes non-typical spherical sterols to accumulate in the fungal cytoplasmic membranes [27].

Amorolfine is effective against dermatophytes (Trichophyton spp., Microsporum spp., Epidermophyton spp.), yeasts (Candida spp., Cryptococcus spp., Malassezia spp.), some molds (Alternaria spp., Hendersonula spp., Scopulariopsis spp.) and other pathogenic fungi (Cladosporium, Coccidioides, Histioplasma, Sporothrix) but not against bacteria except Actinomyces [22, 25] (Table 1).

Concentration of amorolfine increases from $5 \%$ to 27\% with solvent evaporation [26]. Pharmacokinetic properties enable good penetration through the nail to the nail bed and absorption of the active substance into circulation is very low. Amorolfine can be detected in the nail for about 2 weeks; most types of fungi are sensitive to its low concentration [21, 22]. Treatment with amorolfine should continue until clinical and mycological cure is achieved. Time of treatment depends on severity and site of infection as well as growth of nail plate, usually 6-12 months. Assessment of the treatment efficacy is recommended every 3 months [20, 22].

The amorolfine lacquer is applied once or twice a week on a cleaned nail plate and left for 3-5 min until dry. Organic solvents should not be used to remove the preparation. Adverse effects such as erythema, burning sensation, nail discoloration and onycholysis may be observed [28, 29].

In a randomized double-blind study, the effectiveness of amorolfine $2 \%$ (77 patients) to amorolfine 5\% lacquers (80 patients) was evaluated. Patients with toenail onychomycosis were investigated, the lacquers were applied 
Table 1. Amorolfine vs. ciclopirox lacquers: comparison of properties

\begin{tabular}{|c|c|c|}
\hline Parameter & Amorolfine & Ciclopirox \\
\hline Chemical structure & Morpholine derivative - phenyl-morpholine & Pyridine derivative - hydroxy pyridines \\
\hline $\begin{array}{l}\text { Transungual drug delivery } \\
\text { system used in the lacquer }\end{array}$ & Water-insoluble polymers & $\begin{array}{l}\text { Water-insoluble polymers, hydroxypropyl-chitosan } \\
\text { (HPC) }\end{array}$ \\
\hline Mechanism of action & $\begin{array}{l}\text { Inhibits sterol synthesis in fungal cell wall, } \\
\text { depletion of ergosterol and accumulation of } \\
\text { non-typical spherical sterols }\end{array}$ & $\begin{array}{l}\text { Decrease in activity of metal-dependent enzymes } \\
\text { which affects ion transport through cytoplasmatic } \\
\text { membranes }\end{array}$ \\
\hline Antifungal activity & $\begin{array}{l}\text { Dermatophytes (Trichophyton spp., Microsporum } \\
\text { spp., Epidermophyton spp.), yeasts (Candida } \\
\text { spp., Cryptococcus spp., Malassezia spp.), } \\
\text { molds (Alternaria spp., Hendersonula spp., } \\
\text { Scopulariopsis spp.), other pathogenic fungi } \\
\text { (Cladosporium, Coccidioides, Histioplasma, } \\
\text { Sporothrix) }\end{array}$ & $\begin{array}{l}\text { Dermatophytes (Trichophyton spp., Microsporum } \\
\text { spp., Epidermophyton floccosum), yeasts (Candida } \\
\text { spp., Malassezia furfur, Cryptococcus neoformans, } \\
\text { Sacchromyces cerevisiae), molds (Scopulariopsis } \\
\text { brevicaulis, Aspergillus spp., Fusarium solani) }\end{array}$ \\
\hline Antibacterial activity & Only Actinomyces & $\begin{array}{l}\text { Gram-positive bacteria (Staphylococcus spp., } \\
\text { Streptococcus spp.), Gram-negative bacteria } \\
\text { (Escherichia spp., Proteus spp., Klebsiella spp., } \\
\text { Salmonella spp., Shigella spp., Bacillus spp., } \\
\text { Pseudomonas spp.), Mycoplasma }\end{array}$ \\
\hline Anti-inflammatory activity & None & Present \\
\hline
\end{tabular}

daily for 6 months, then after 3 months the effect of the treatment was assessed. Mycological cure was obtained in 55\% (negative culture and negative microscopic examination) using amorolfine $2 \%$ and $60 \%$ patients treated with amorolfine $5 \%$. Complete cure, defined as no mycological findings and complete clinical cure or maintenance $<10 \%$ of the involved nail, was achieved in $12 \%$ of patients treated with amorolfine $2 \%$ and $38 \%$ of patients with amorolfine 5\% [30].

The aim of another multicentre randomized study, carried out on 317 patients, was to evaluate the efficacy of amorolfine 5\% applied once or twice a week for 6 months. Three months after discontinuation of the therapy $71 \%$ of patients using the agent once a week had a negative result of mycological investigation compared to $76 \%$ of those applying it twice a week. Clinical and mycological cure (according to the criteria mentioned above) was achieved in $46 \%$ and $51.8 \%$ of patients, respectively [31].

Similar results were obtained by Zaug and Bergstraesser in the study on 725 patients with toenail onychomycosis treated with amorolfine once or twice a week. Complete cure (mycological cure and clinical improvement with $<10 \%$ nail involvement) was achieved in $44.6 \%$ of patients [32].

\section{Ciclopirox}

Ciclopirox is a hydroxy-pyridone derivative. It has been investigated since 1973 but used in the form of lacquer since the 1990s. Ciclopirox is marketed in many forms (cream, suspension, shampoo, gel, solution, powder, globules) and used for treatment of skin, scalp, onycho- mycosis. It is also used in seborrhoeic dermatitis, pityriasis versicolor, vagina candidiasis [33, 34]. In nail lacquers, ciclopirox is free acid whereas in other formulations it is ethanolamine salt. Preparations with free acid are more active than those with salt $(1: 0.77)[33,35]$. Ciclopirox exerts its anti-fungal activity by chelating trivalent cations, including $\mathrm{Fe}^{3+}$ and $\mathrm{Al}^{3+}$, which results in the inhibition of metal-dependent enzymes, particularly cytochromes, catalases, peroxidases leading to reduced transport of ions through pathogen cytoplasmatic membranes and reduced nutrient intake. Ciclopirox inhibits permeability of aminoacids, e.g. leucine, into a cell, causes loss of potassium ions and inhibits arachidonic acid cascade [33, 34].

Ciclopirox demonstrates a broad antifungal activity. It is active against dermatophytes (Trichophyton spp., Microsporum spp., Epidermophyton floccosum), yeasts (Candida spp., Malassezia furfur, Cryptococcus neoformans, Sacchromyces cerevisiae), molds (Aspergillus spp., Scopulariopsis brevicaulis, Fusarium solani). It has also been shown to be active against Gram-positive bacteria (Trichophyton spp., Microsporum spp., Epidermophyton floccosum), Gram-negative (Escherichia spp., Proteus spp., Klebsiella spp., Salmonella spp., Shigella spp., Bacillus spp., Pseudomonas spp.) and Mycoplasma, Trichomonas vaginalis and other. Antibacterial activity is particularly beneficial in the treatment of mixed infections. The agent has also anti-inflammatory activity by inhibiting arachidonic acid cascade thus inhibiting synthesis of prostaglandins and leukotrienes in polynuclear granulocytes $[33,34]$. In the anti-inflammatory test, ciclopirox has been found to be the most effective compared with naftifine, terbinafine, ketoconazole and econazole [36] (Table 1). 
Ciclopirox, based on water-insoluble polymers, the $8 \%$ concentration increases to about 35\% after application [37]. Systemic absorption is very low. It penetrates well into mycotic nail keratin. Twenty-four hours after onetime application, the concentration significantly exceeds minimal inhibitory concentration (MIC) for Trichophyton rubrum, T. mentagrophytes and Candida albicans [38]. The treatment should be continued until clinical and mycological cure is achieved, usually for about 6-12 months. Four weeks after discontinuation of the therapy, a mycological test should be performed to confirm the cure and avoid activity of possible remains of active substance.

Ciclopirox, with water-insoluble polymers, is applied on the cleaned nail every second day for the first month, so the active substance penetrates the nail, twice a week for the second month and once a week from the third month on. No statistically significant difference in the drug effectiveness between this sequence and daily application was found, however, in most clinical trials daily application was used [39]. Once a week ciclopirox should be removed with cosmetic nail remover. Ciclopirox with hydroxypropyl chitosan is applied daily for 6 h. Hydroxypropyl chitosan preparation is usually applied for the night and washed with water in the morning [24]. Adverse effects of ciclopirox with both vehicles were rarely reported and include itching, scaling, and local burning sensation [21, 22].

Two double-blind, vehicle controlled, randomized studies carried out in the US on 460 patients with mild to moderate distal subungual onychomycosis caused by dermatophytes (20-65\% nail involvement) showed a high safety profile and effectiveness of ciclopirox monotherapy. The agent was applied daily on all toenails, including the healthy ones. The study was conducted for 48 weeks, every 12 weeks direct examination and mycological evaluation were performed. After 48 weeks of treatment, in study I negative culture and negative direct examination were achieved in $29 \%$ of patients compared to $11 \%$ in the placebo group; in study II $-36 \%$ vs. $9 \%$. In study I, clinical cure $(<10 \%$ involvement of the nail plate, negative culture and negative direct examination) was achieved in $6.5 \%$ of patients while in study II - in 12\% [40].

The results of meta-analysis carried out by the same authors in Europe, Asia and South Africa confirmed high efficacy and safety of ciclopirox by mycological evaluation $52.6 \pm 4.2 \%$ (46.7-85.7\%). The studies were conducted for 6-12 months; patients infected with Candida and those with affected both toes and fingers were also included in the study, frequency of ciclopirox lacquer application was also different, which may have caused differences in efficacy of the treatment in the US and outside $[40,41]$.

Monti et al. [42] compared fingernail penetration of ciclopirox with HPCH vehicle to standard amorolfine 5\% lacquer applied twice a week. The volunteers applied lacquer with HPCH on fingernails of one hand and reference amorolfine preparation on the other. At baseline and after 15 and 25 days efficiency coefficients, as ratios of nail concentration and minimum mycostatic concentration, for T. rubrum and C. parapsilosis were calculated. Ciclopirox with HPCH vehicle exhibited significantly higher efficacy for both species compared to amorolfine reference.

\section{Combination therapy}

Combined therapy of oral and topical drugs is a beneficial and commonly used therapeutic method due to the fact that monotherapy with topical drugs is often not fully effective and also risk of adverse effects as well as interactions with oral drugs taken for a long time. Combined therapy uses synergism of antifungal drugs with different mechanisms of action [21, 43].

Several studies were carried out to evaluate efficacy of combined therapy of amorolfine and ciclopirox lacquers with antifungal oral drugs. Ciclopirox lacquer combined with terbinafine and itraconazole was found to increase the percentage of cured patients [44, 45]. An increase in efficacy of combination of amorolfine lacquer and terbinafine, itraconazole, griseofulvin and fluconazole was also observed [46]. The data indicate that to obtain the therapeutic effect, the intake of a systemic drug combined with amorolfine is shorter compared to monotherapy with oral drugs [47-49].

Long-term use of amorolfine lacquer, once every 2 weeks, was found to be effective in prophylaxis of onychomycosis recurrence in patients earlier treated with terbinafine inmonotherapy or combined with amorolfine [50].

\section{Conclusions}

Topical preparations in the form of lacquers applied on the nail plate are a valuable tool in the treatment of onychomycosis, however, monotherapy should be limited. It is difficult to assess efficacy of amorolfine and ciclopirox in monotherapy due to the different criteria of recruiting patients for investigations, different study periods and post-treatment assessment. Combined therapy of antifungal preparations in the form of lacquers and oral drugs such as terbinafine or itraconazole increases efficacy of the treatment and shortens duration of oral antifungal drug intake.

\section{Conflict of interest}

The authors declare no conflict of interest.

\section{References}

1. Faergemann J, Baran R. Epidemiology, clinical presentation and diagnosis of onychomycosis. Br J Dermatol 2003; 149: $1-4$.

2. Hay R. Literature review. Onychomycosis. J Eur Acad Dermatol Venereol 2005; 19: 1-7. 
3. Roberts DT. Prevalence of dermatophyte onychomycosis in the United Kingdom: results of an omnibus survey. Br J Dermatol 1992; 126: 23-37.

4. Heikkala H, Stubbs S. The prevalence of onychomycosis in Finland. Br J Dermatol 1995; 133: 699-701.

5. Effendy I, Lecha M, Feuilhade de Chauvin M, et al. European Onychomycosis Observatory: epidemiology and clinical classification of onychomycosis. J Eur Acad Dermatol Venereol 2005; 19: 8-12.

6. Adamski Z, Batura-Gabryel H. (ed.) Medical mycology for doctors and students. Medical Publishing House of Poznan University of Medical Sciences, Poznan 2007; 98-109.

7. Baran E, Szepietowski J, Walów B, et al. Fungal skin infections in the Lower-Silesian region in the years 1974-1991: part II, localization of skin lesions. Przegl Dermatol 1993; 80: 49-58.

8. Roberts DT. Onychomycosis: current treatment and future challenges. Br J Dermatol 1999; 141: 1-4.

9. Sikora M, Pachołek T, Soter K, et al. Analysis of fungal skin and skin appendages infections in the region of Wrocław in the years 1995-1999. Mikol Lek 2000; 7: 145-51.

10. Szepietowski JC, Reich A, Garłowska E, et al. Factors influencing coexistence of toenail onychomycosis with tinea pedis and other dermatomycoses: a survey of 2761 patients. Arch Dermatol 2006; 142: 1279-84.

11. Elewski BE. Onychomycosis: pathogenesis, diagnosis, and management. Clin Microbiol Rev 1998; 11: 415-29.

12. Gupta AK, Uro M, Cooper EA. Onychomycosis therapy: past, present, future. J Drugs Dermatol 2010; 9: 1109-13.

13. Lecha M, Effendy I, Feuilhade de Chauvin M, et al. Taskforce on Onychomycosis Education. Treatment options - development of consensus guidelines. J Eur Acad Dermatol Venereol 2005; 19: 25-33.

14. Hardjoko FS, Widyanto S, Singgih I, et al. Treatment of onychomycosis with a bifonazole-urea combination. Mycoses 1990; 33: 167-71.

15. Lecha V, Martinez C, Mascaro JM. Experience with topical application of bifonazole plus urea in the treatment of onychomycosis. J Dermatol Treat 1990; 1: 131-2.

16. Szepietowski JC, Reich A. Combined treatment of toenail onychomycosis with terbinafine and amorolfine - prospective open-label study. Dermatol Klin 2008; 10: 67-71.

17. Baran E, Adamski Z, Maleszka R, et al. Combination therapy - recommendations for severe onychomycosis. Mikol Lek 2003; 10: 75-8.

18. Thomas J, Jacobson GA, Narkowicz CK, et al. Toenail onychomycosis: an important global disease burden. J Clin Pharm Therapeut 2010; 35: 497-519.

19. Grover C, Khurana A. An update on treatment of onychomycosis. Mycoses 2012; 55: 541-51.

20. Iorizzo M, Piraccini BM, Tosti A. Today's treatment options for onychomycosis. J Dtsch Dermatol Ges 2010; 8: 875-9.

21. Baran R, Kaoukhov A. Topical antifungal drugs for the treatment of onychomycosis: an overview of current strategies for monotherapy and combination therapy. J Eur Acad Dermatol Venereol 2005; 19: 21-9.

22. Finch JJ, Warshaw EM. Toenail onychomycosis: current and future treatment options. Dermatol Ther 2007; 20: 31-46.

23. Dominicus R, Weider C, Tate H, et al. Open-label study of the efficacy and safety of topical treatment with TDT 067 (terbinafine in Transfersome) in patients with onychomycosis. Br J Derm 2012; 166: 1357-80.

24. Baran R, Tosti A, Hartmane I, et al. An innovative water-soluble biopolymer improves efficacy of ciclopirox nail lacquer in the management of onychomycosis. J Eur Acad Dermatol Venereol 2009; 23: 773-81.

25. Gupta AK, Ryder JE, Baran R. The use of topical therapies to treat onychomycosis. Dermatol Clin 2003; 21: 481-9.

26. Marty JP. Amorolfine nail lacquer: a novel formulation. J Eur Acad Dermatol Venereol 1995; 5: 17-21.

27. Polak AM. Preclinical data and mode of action of amorolfine. Clin Exp Dermatol 1992; 17: 8-12.

28. Rigopoulos D, Katsambas A, Antoniou C, et al. Discoloration of the nail plate due to the misuse of amorolfine $5 \%$ nail lacquer. Acta Derm Venereol 1996; 76: 83-4.

29. Kim BJ, Ro BI. Hyperpigmentation due to the overuse of amorolfine nail lacquer. Korean J Med Mycol 2002; 7: 224-6.

30. Lauharanta J. Comparative efficacy and safety of amorolfine nail lacquer 2\% versus 5\% once weekly. Clin Exp Dermatol 1992; 17: 41-3.

31. Reinel D, Clarke C. Comparative efficacy and safety of amorolfine nail lacquer 5\% in onychomycosis, once-weekly versus twice-weekly. Clin Exp Dermatol 1992; 17: 44-9.

32. Zaug M, Bergstraesser M. Amorolfine in the treatment of onychomycoses and dermatomycoses (an overview). Clin Exp Dermatol 1992; 17: 61-70.

33. Subissi A, Monti D, Togni G. Ciclopirox recent nonclinical and clinical data relevant to its use as a topical antimycotic agent. Drugs 2010; 70: 2133-52.

34. Gupta AK, Plott T. Ciclopirox: a broad-spectrum antifungal with antibacterial and anti-inflammatory properties. Int J Dermatol 2004; 43 Suppl. 1: 3-8.

35. Kołodziej T, Białynicki-Birula R. Ciclopirox in the treatment of seborrhoeic dermatitis. Mikol Lek 2004; 11: 329-32.

36. Rosen T, Schell BJ, Orengo I. Anti-inflammatory activity of antifungal preparations. Int J Dermatol 1997; 36: 788-92.

37. Bohn M, Kraemer KT. Dermatopharmacology of ciclopirox nail lacquer topical solution $8 \%$ in the treatment of onychomycosis. J Am Acad Dermatol 2000; 43: 57-69.

38. Bohn M, Kraemer KT. Dermatopharmacology of ciclopirox nail lacquer topical solution $8 \%$ in the tretment of onychomycosis. J Am Acad Dermatol 2000; 43 (4 Suppl.): S57-69.

39. Schalka S, Nunes S, Gomes Neto A. Comparative clinical evaluation of efficacy and safety of a formulation containing ciclopirox $8 \%$ in the form of a therapeutic nail lacquer in two different posologies for the treatment of onychomycosis of the toes. An Bras Dermatol 2012; 87: 19-25.

40. Gupta AK, Fleckman P, Baran R. Ciclopirox nail lacquer topical solution $8 \%$ in the treatment of toenail onychomycosis. J Am Acad Dermatol 2000; 43: S70-80.

41. Gupta AK, Joseph WS. Ciclopirox $8 \%$ nail lacquer in the treatment of onychomycosis of the toenails in the United States. J Am Podiatric Med Assoc 2000; 90: 495-501.

42. Monti D, Herranz L, Bo D, et al. Nail penetration and predicted mycological efficacy of an innovative hydrosoluble ciclopirox nail lacquer vs. a standard amorolfine lacquer in healthy subjects. J Eur Acad Dermatol Venereol 2013; 27 : e153-8.

43. Evans $\mathrm{E}$. The rationale for combination therapy. Br J Dermatol 2001; 145: 9-13.

44. Nolting S. Open studies of ciclopirox nail lacquer in onychomycosis: a review. In: Hydroxy-pyridones as antifungal agents with special emphasis on onychomycosis. Shuster S (ed.). Springer-Verlag, Berlin 1999; 75-80.

45. Avner S, Nir N, Henri T. Combination of oral terbinafine and topical ciclopirox compared to oral terbinafine for the treatment of onychomycosis. J Dermatolog Treat 2005; 16 : 327-30. 
46. Sigurgeirsson B, Olafsson JH, Steinsson JT, et al. Efficacy of amorolfine nail lacquer for the prophylaxis of onychomycosis over 3 years. J Eur Acad Dermatol Venereol 2010; 24: 910-5.

47. Baran R, Feuilhade M, Combernale P. A randomized trial of amorolfine $5 \%$ solution nail lacquer combined with oral terbinafine compared with terbinafine alone in the treatment of dermatophytic toenail onychomycoses affecting the matrix region. Br J Dermatol 2000; 142: 1177-83.

48. Baran R, Sigurgeirsson B, de Berker D, et al. A multicentre, randomized, controlled study of the efficacy, safety and cost-effectiveness of a combination therapy with amorolfine nail lacquer and oral terbinafine compared with oral terbinafine alone for the treatment of onychomycosis with matrix involvement. Br J Derm 2007; 157: 149-57.

49. Rigopoulos D, Katoulis AC, Ioannides D. A randomized trial of amorolfine $5 \%$ solution nail lacquer in association with itraconazole pulse therapy compared with itraconazole alone in the treatment of Candida fingernail onychomycosis. Br J Derm 2003; 149: 151-6.

50. Sigurgeirsson B, Olafsson JH, Steinsson JT, et al. Efficacy of amorolfine nail lacquer for the prophylaxis of onychomycosis over 3 years. J Eur Acad Dermatol Venereol 2010; 24: 910-5. 